\title{
Archéopages
}

Archéopages

Archéologie et société

42 | 04-07/2015

Construire en terre crue

\section{Fortifier en terre crue au XVII siècle}

L'escarpe maçonnée du Fort Saint-Sébastien de Saint-Germain-en-Laye An earthen fortification of the seventeenth century. The Fort Saint-Sébastien's escarpment at Saint-Germain-en-Laye

Fortificar con barro en el siglo XVII. La escarpa mamposteada del Fuerte SaintSébastien de Saint-Germain-en-Laye

\section{Séverine Hurard et Gwenaël Mercé}

\section{(2) OpenEdition}

\section{Journals}

Édition électronique

URL : https://journals.openedition.org/archeopages/1298

DOI : $10.4000 /$ archeopages. 1298

ISSN : 2269-9872

\section{Éditeur}

INRAP - Institut national de recherches archéologiques préventives

Édition imprimée

Date de publication : 1 mars 2016

Pagination : 106-115

ISSN : 1622-8545

\section{Référence électronique}

Séverine Hurard et Gwenaël Mercé, « Fortifier en terre crue au xvı" siècle », Archéopages [En ligne], 42 | 04-07/2015, mis en ligne le 01 juillet 2017, consulté le 02 juin 2021. URL : http://

journals.openedition.org/archeopages/1298; DOI : https://doi.org/10.4000/archeopages.1298 


\section{Fortifier en terre crue au xvII ${ }^{e}$ siècle L'escarpe maçonnée du Fort Saint-Sébastien de Saint-Germain-en-Laye}

Séverine Hurard Inrap, UMR 7041 «ArScan - Archéologies et Sciences de lantiquité" "

avec la collaboration de Gwenaël Mercé Inrap

\section{6}

1. Fouille menée entre 2011 et 2012 sur une surface de 28 hectares, sous la direction de Séverine Hurard, Inrap.
La fouille ${ }^{\mathbf{1}}$ du Fort Saint-Sébastien a constitué une fenêtre d'observation inédite sur la société des gens de guerre du dernier tiers du XVII ${ }^{\mathrm{e}}$ siècle (Hurard, Rochart, 2013 ; Hurard, Lorin, Tixador, 2014; Hurard, 2014; Hurard, Rochart, 2015). Au cœur de la région parisienne, le fort montre le large investissement concédé à la préparation à la guerre de siège dans laquelle s'engagent Louis XIV et son état-major à la fin des années 1660 [ill. 1]. Ouvrage de campagne non pérenne et camp retranché en appui sur la Seine, le « Fort Saint-Sébastien » est opérationnel d'avril à septembre 1669. Il est ensuite rasé et rebouché pour les besoins de la construction, en 1670, d'une ligne de circonvallation de $4 \mathrm{~km}$, le « Camp de SaintSébastien » [ill. 2].

Cette condamnation/remplacement du premier ouvrage fortifié a favorisé l'excellente conservation d'une remarquable escarpe de fossé, revêtue d'une maçonnerie de terre crue, dont on ne connaît aujourd'hui aucun autre équivalent en Europe pour la période. La technique de la maçonnerie en terre appliquée à la fortification moderne semble aujourd'hui sous-estimée. Si aucun équivalent au Fort Saint-Sébastien n'a pu être mis en évidence jusqu'ici, il semble qu'il s'agisse surtout d'un effet de sources. La tradition de la construction en mottes de gazon est en effet connue depuis l'Antiquité - Pline la mentionne spécifiquement pour le retranchement des camps (Guillaud, 2003) et encore utilisée par le génie militaire au début de la première guerre mondiale pour renforcer les parapets de tranchée. La découverte de l'escarpe maçonnée de terre crue du Fort Saint-Sébastien fait ainsi ressurgir une technique dont les auteurs modernes de traités de fortifications se font également l'écho, quoique de manière plus anecdotique que pour la fortification de pierre ou de brique. Elle témoigne du soin apporté à la construction d'une fortification de campagne éphémère et donne la mesure des investissements techniques et logistiques d'un chantier de terrassement de la guerre de siège qui, aux portes de Paris, en temps de paix, mobilise d'importantes ressources matérielles et humaines, répétition à échelle réduite des opérations menées dans les Flandres ou aux Pays-Bas sous le règne de Louis XIV.

Un camp retranché de terre, de bois... et de terre crue maçonnée

Le Fort Saint-Sébastien est une fortification de campagne de forme simple. Le front sud, fouillé intégralement, mesure $660 \mathrm{~m}$ de long d'un bastion à l'autre. Il est hérissé de deux bastions, de deux redans flanquant la porte et sa demi-lune. La confrontation des données archéologiques avec les données textuelles et planimétriques permet de proposer l'hypothèse d'un fort rectangulaire de $590 \mathrm{~m}$ de long du nord au sud, composé de quatre bastions d'angle, de huit redans, flanqués de quatre portes. Il atteindrait donc une surface de 39 hectares dont seulement 11 ha ont été fouillés (fossés compris), soit un quart de la surface totale. Le fossé de cet ouvrage fortifié a pu être fouillé intégralement sur une longueur linéaire de $1125 \mathrm{~m}$ (total estimé à $2740 \mathrm{~m}$, incluant la longueur des faces et des flancs des redans et bastions). L'état de conservation général du terrain permet de penser que le fossé de $7 \mathrm{~m}$ de large pour $3 \mathrm{~m}$ de profondeur est conservé dans la quasi-totalité de son creusement.

L'escarpe du fossé a fait l'objet d'un soin particulier tant lors de la construction qu'au moment de la fouille. Elle était en effet intégralement revêtue d'une remarquable maçonnerie de terre crue, se déployant sur toute sa hauteur [ill. 3]. La restitution du rempart et des élévations de terre 


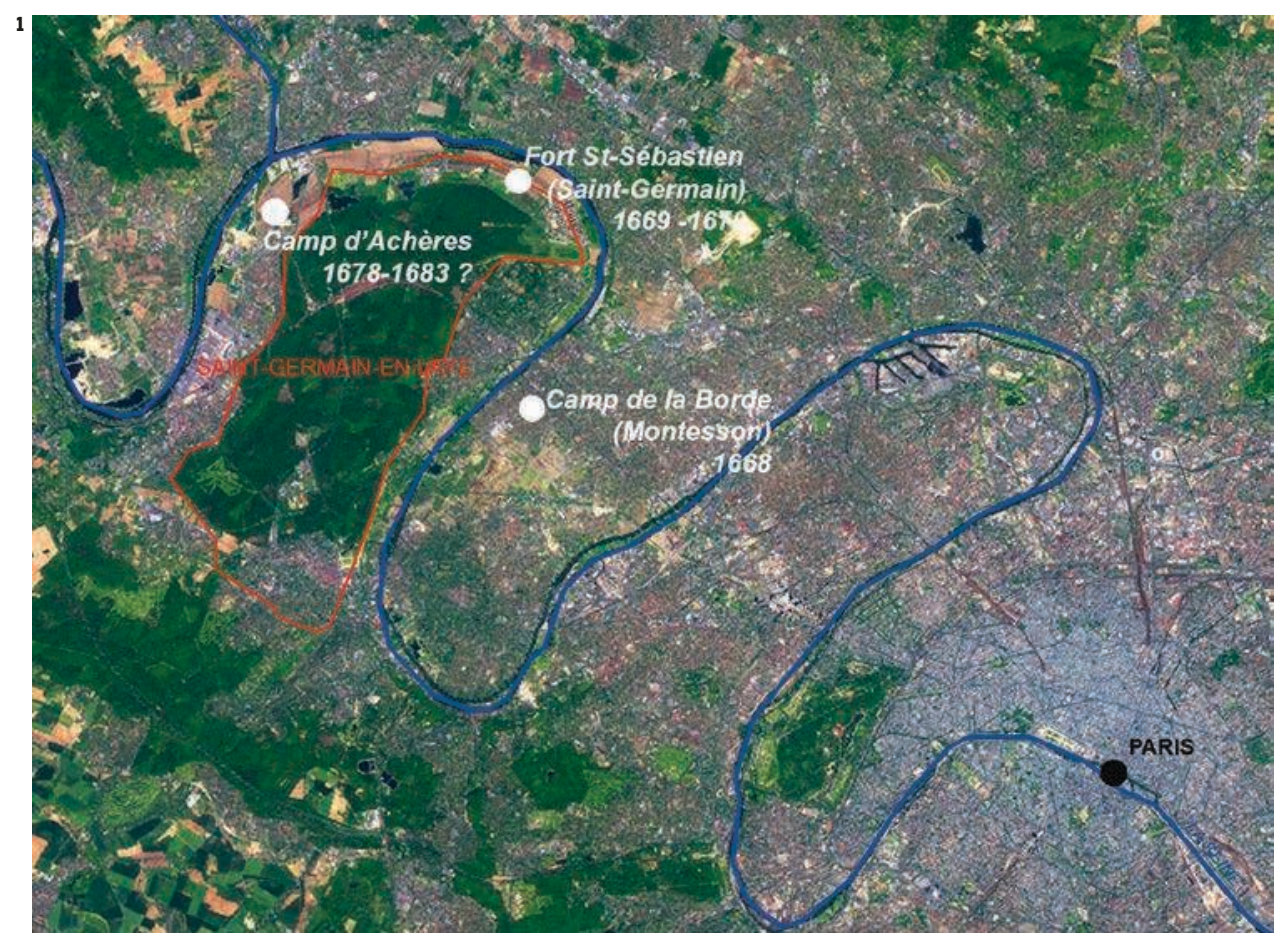

1. La plaine alluviale dite d'Achères, formée par la

Seine, est située à une

trentaine de kilomètres à

l'ouest de Paris. Elle

Saint-Sébastien

correspondent à deux

connut entre 1668 et 1683

ouvrages militaires et

leurs campements

trois camps militaires qui succédèrent lors de deux

permirent à Louis XIV

d'assurer la préparation de

campagnes réunissant les

ses troupes, pendant

troupes de la maison

chaque courte période de

militaire du roi au cours

paix, entre la guerre de et 1670 . Ils ont été fouillés

Dévolution, la guerre de à hauteur de 1/4 et1/10

Hollande et la guerre des de leur surface totale.

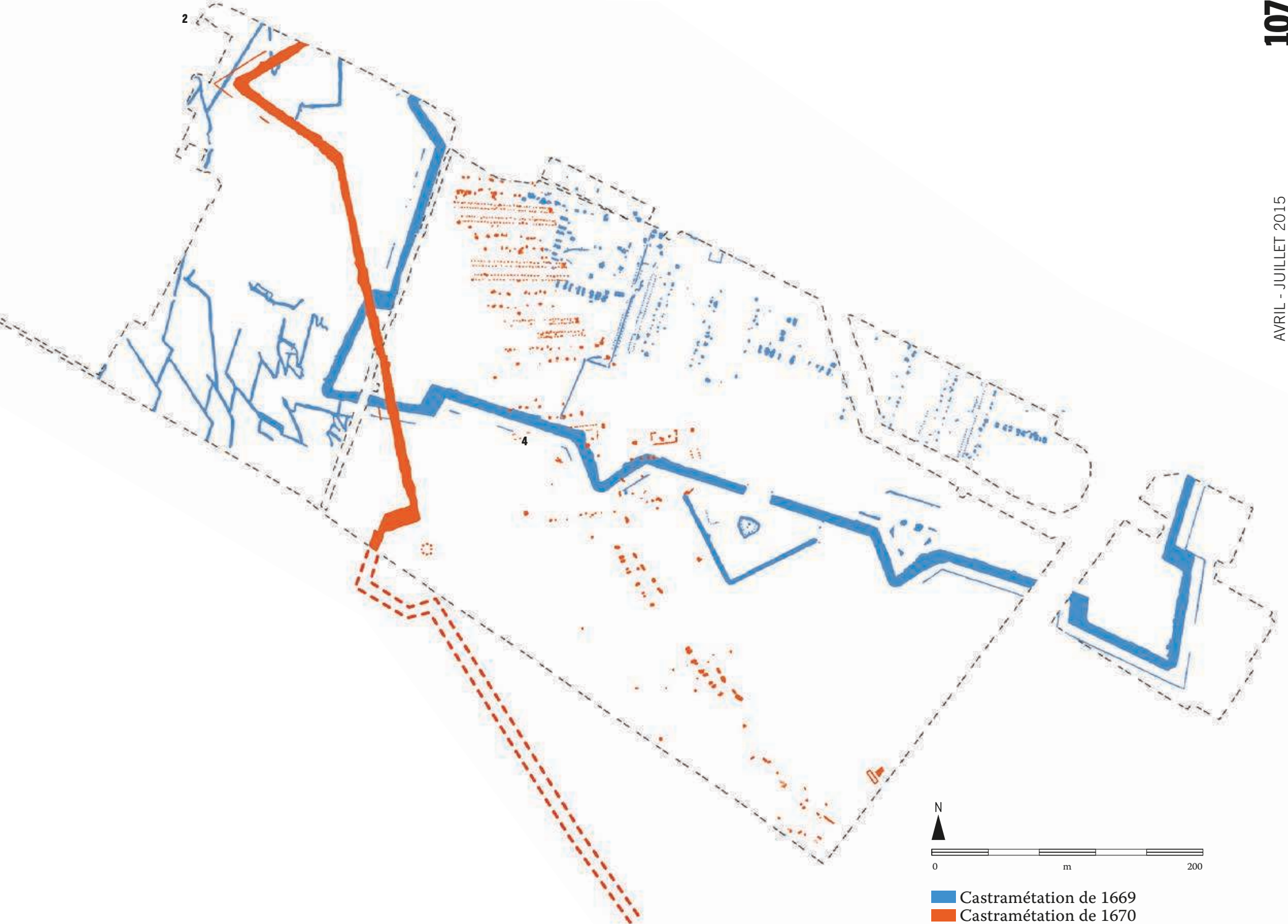




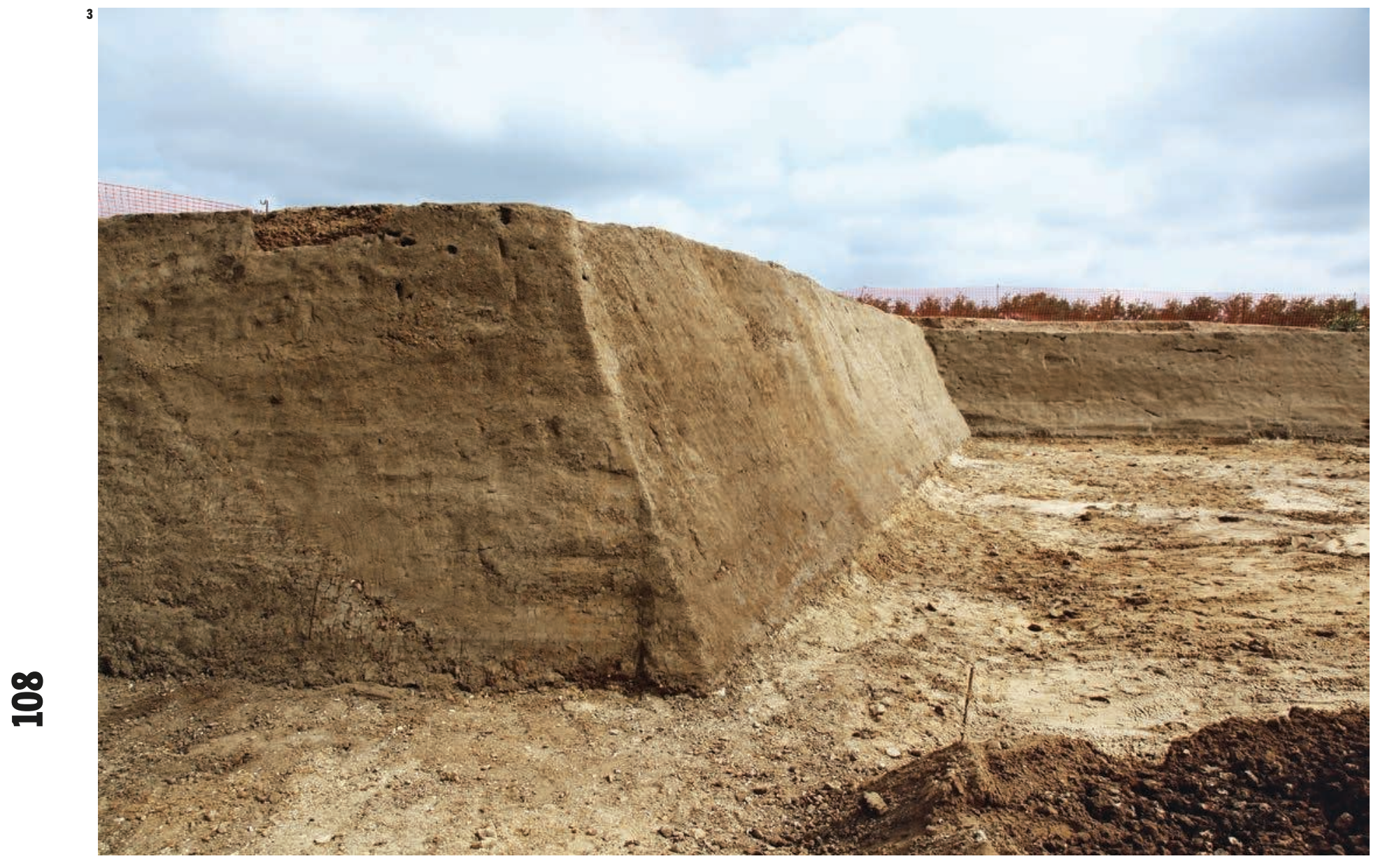

\section{$4 \quad$ Banquette}

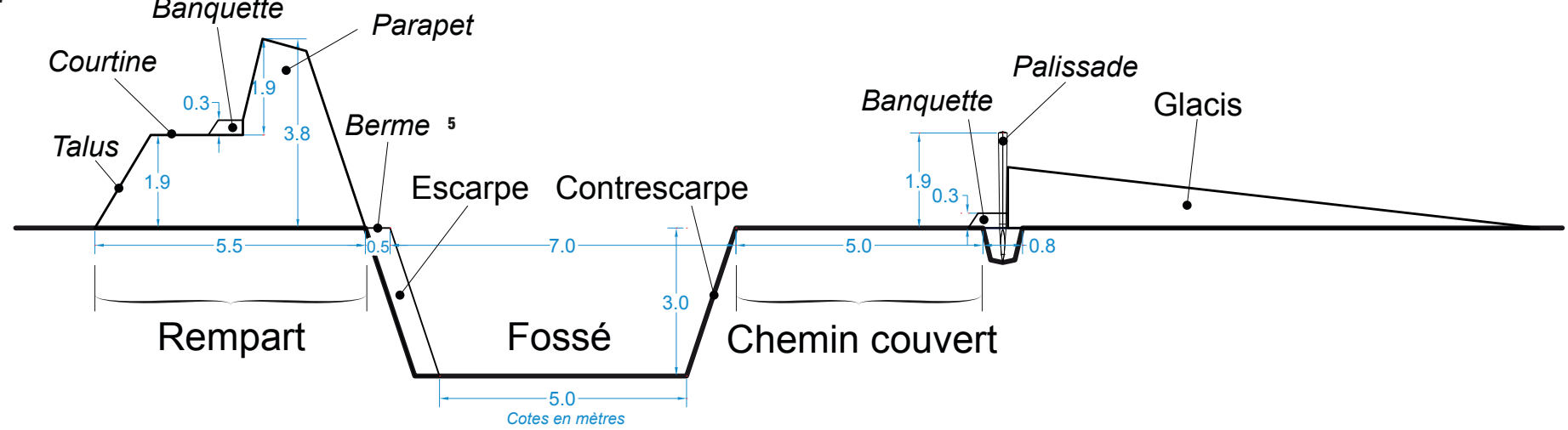

4. Profil restitué de

l'ouvrage fortifié. Le

rempart devait être une

masse de terre de 5 à $6 \mathrm{~m}$

de large à la base, pour une

hauteur totale de 3 à $4 \mathrm{~m}$,

incluant courtine et

parapet. La hauteur du

parapet est estimée à

environ $1,95 \mathrm{~m}$, sa base

étant équivalente à un

quart de la largeur du

rempart, soit 1,30 m.

est directement posé sur

substrat sableux et n'est

associé à aucun dispositif

de fondation. 


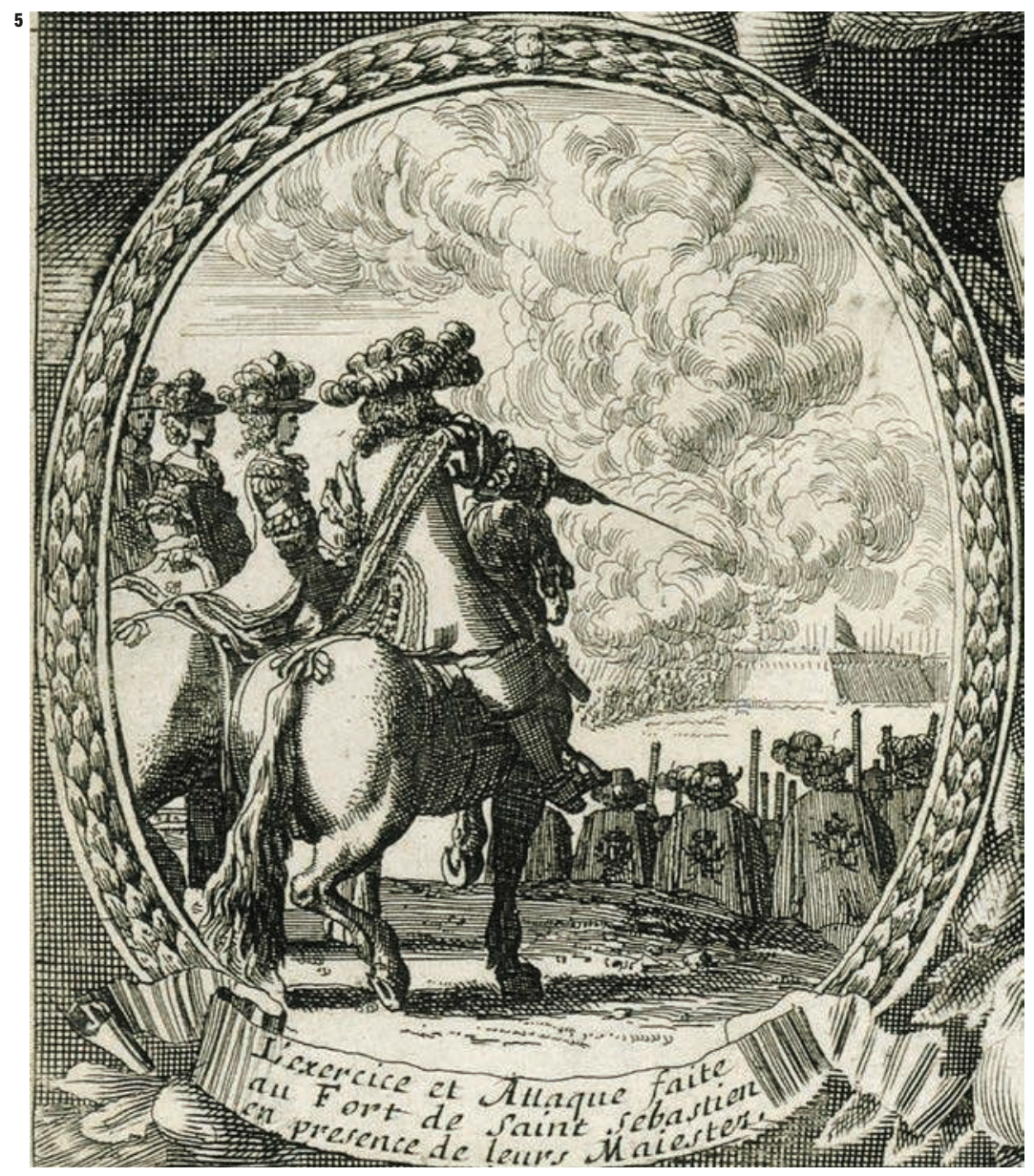

5.Assaut mené au Fort Saint-Sébastien en présence de la famille royale, représentant l'angle sud-ouest du rempart de l'ouvrage de 1669. Soumis à l'assaut des fantassins et piquiers, le rempart est enveloppé d'un épais nuage de fumée. Une rangée de pieux, placée au deux tiers de la hauteur du rempart, est fichée presque

horizontalement dans la masse de terre. (Médaillon du calendrier-estampe de l'almanach de 1670 :

« l'Exercice et Attaque faîte du Fort de Saint-Sebastien en presence de leur Maiestez )).

2. La hauteur d'assise de $10 \mathrm{~cm}$ multipliée par la largeur de $24 \mathrm{~cm}$ donne une surface externe de $240 \mathrm{~cm}^{2}$. Celle-ci divisée par 2 puis multipliée par la profondeur moyenne de $28 \mathrm{~cm}$ permet d'aboutir à un volume par prisme de $3360 \mathrm{~cm}^{3}$.

3. $60 \times 3300 \mathrm{~cm}^{3}=$

$198000 \mathrm{~cm}^{3}=0,198 \mathrm{~m}$ 4. Réalisée par Cécilia

Cammas, Inrap.

5. Réalisée par David

Gandreau au Craterre de Grenoble. placées au-dessus de l'escarpe propose de ne faire monter cette maçonnerie qu'à hauteur de la « campagne », c'est-à-dire au sommet de la contrescarpe. La quasi-absence de modules de terre crue rejetés dans le comblement du fossé au moment du rebouchage nous incite à cette hypothèse par ailleurs étayée par une des propositions illustrées par Vauban dans son traité de la défense des places. Le dispositif pouvait être complété de fascines et de cordons de bois [ill. 4]. La gravure de l'almanach de 1670 représente un rempart surmonté à son sommet d'une fraise de fortification en bois tout à fait cohérente avec les usages [ill. 5]. A contrario, la contrescarpe ne semble faire l'objet d'aucun traitement particulier. Aucune trace n'a mis en évidence l'existence de terre crue maçonnée, ou simplement plaquée, ni celle de placage de planches ou de fascines, attestée dans des exemples diachroniques.

\section{La mise à nue d'une technique de maçonnerie}

Les écorchés réalisés dans le matériau révèlent une maçonnerie à appareil irrégulier et assises régulières. Ces dernières mesurent en moyenne une dizaine de centimètres de hauteur [ill. 6]. De face, l'ensemble se compose de prismes rectangulaires de dimensions diverses. Les coupes stratigraphiques réalisées dans le massif montrent en revanche clairement que ces éléments sont de section triangulaire et forment une masse dentelée.
On compte en moyenne cinq modules par assise sur une portion de $1 \mathrm{~m}$ de large et 12 assises pour une section de $1 \mathrm{~m}$ de haut, soit 60 mottes pour $1 \mathrm{~m}^{2}$. En volume $\mathrm{z}^{2}$, un prisme d'argile représente environ $3300 \mathrm{~cm}^{3}$. Chaque mètre carré nécessite donc environ $0,2 \mathrm{~m}^{3}$ de matériau ${ }^{3}$ [ill. 7]. Le massif de maçonnerie est constitué d'une combinaison de terre crue, placée en revêtement, et d'une structure de sable grossier, assurant la cohésion et la résistance du massif à l'érosion et aux dégradations naturelles et/ou mécaniques [ill. 8]. L'épaisseur générale du massif est très en deçà des recommandations faites par Vauban dans le Traité de l'attaque et de la deffense des places (Vauban, 17..a) où il précise, pensant à des ouvrages fortifiés d'une autre dimension, que l'épaisseur du mur devra être proportionnelle à la hauteur de la maçonnerie et pourra atteindre 8 pieds, c'est-à-dire 2,40 m. À Saint-Germain, simple redoute de campagne, le massif atteint seulement de o,50 à $1 \mathrm{~m}$.

Plusieurs échantillons de ce matériau ont été soumis à une étude micromorphologique et à une analyse de matériaux ${ }^{5}$. Les deux études montrent que les matériaux prélevés sont des limons carbonatés d'origine alluviale, prélevés sous la couche végétale, dans un horizon dépourvu de racines et de bioturbations. Le matériau est fortement cohésif et moyennement expansif. Il absorbe l'eau de manière lente, sans aucune dégradation apparente, et présente une très bonne résistance à l'abrasion. Il n'a fait l'objet d'aucun ajout végétal ou d'aucun agrégat résiduel malaxé et d'aucun façonnage ou mise en gabarit au moment de la mise en œuvre. L'extraction de la terre s'est apparemment faite en « mottes » par un objet coupant. Celles-ci ont été taillées en forme de dents et ensuite retaillées en surface pour égaliser et donner un aspect plus uniforme. Tout semble donc indiquer que le matériau utilisé ne présente pas de couvert végétal. La cohésion du matériau seul semble suffisante à assurer la solidité de la construction, a fortiori si les ingénieurs prennent en considération la courte durée d'utilisation du camp. La lecture des traités nous amène à questionner le sens du terme « gazon » et à nous demander si, par métonymie, le terme ne désigne pas de manière générale la technique du revêtement à la terre, plutôt que la présence réelle d'herbe ou de végétation à la surface du revêtement.

Les traités de fortification modernes (Marolois, 1615-1616 ; Ville, 1628 ; Fournier, 1648), préconisent que les mottes de gazons ou argiles grises soient grasses pour résister aux pluies et à la chaleur. Ces gazons à « revêtir les terrasses » y sont toujours composés d'éléments triangulaires où l'épaisseur de la face est six fois plus importante que la queue : « il doit avoir 3 ou 4 pouces d'épaisseur, sept à 8 de largeur, $\mathcal{E} 12$ à 15 de long, E doit diminuer d'épaisseur vers la queue » (La Fontaine, 1666). La planche d'illustration de l'ouvrage de Fournier montre qu'une armature de pieux de bois peut permettre de stabiliser le parement. Antoine de 


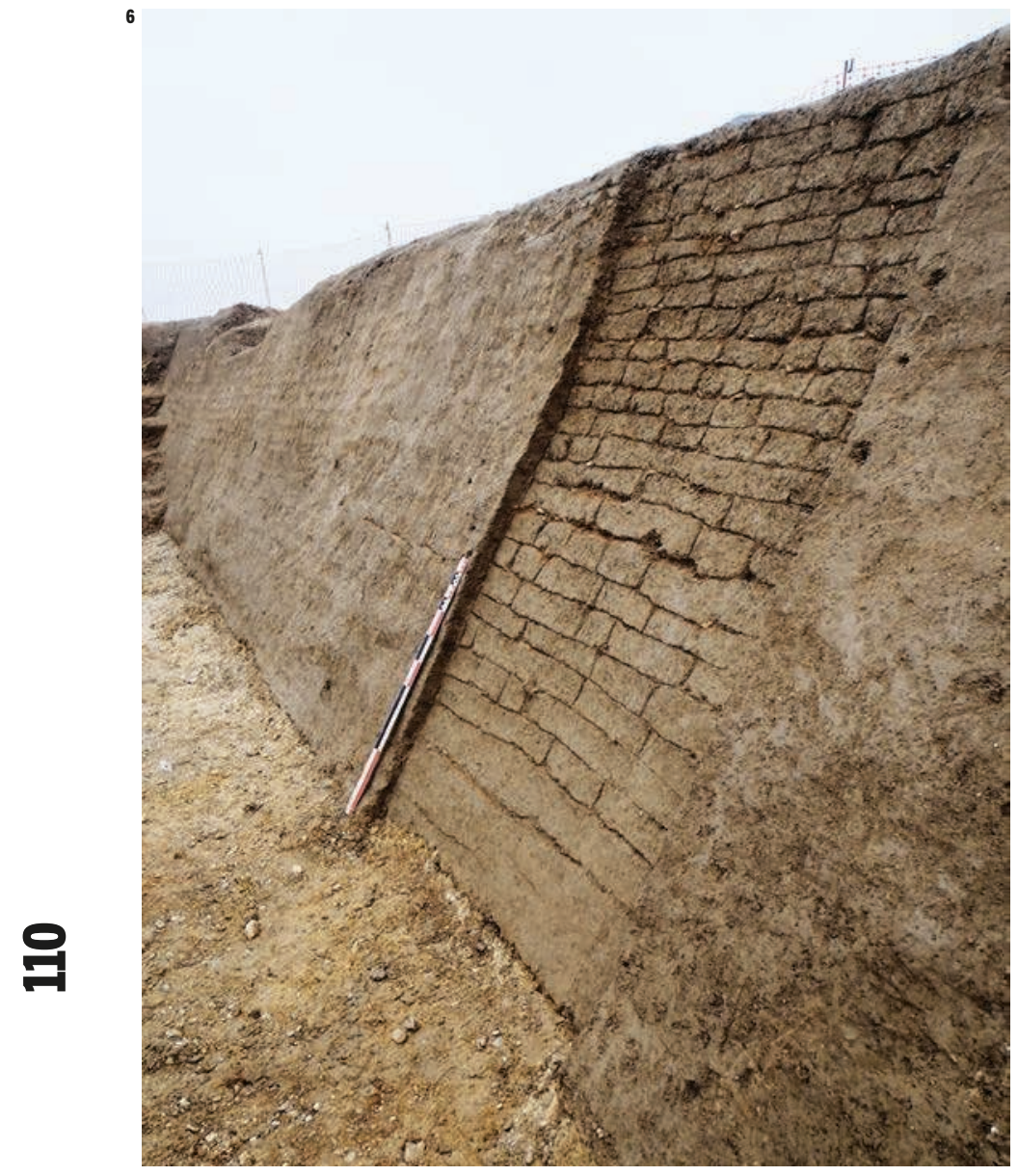

6.Vue d'une section de

l'escarpe et écorché

réalisé dans la maçonnerie

Structurée en assises

régullières, la maçonnerie

présente un appareil

irrégulier où la largeur des

modules oscille entre 25 et

$80 \mathrm{~cm}$.

La terre crue exploitée à

l'état humide se prête

sans difficultés à un

chaînage grossier des

blocs dont témoignent les

nombreuses déformations

des modules dans les

angles des bastions et

redans.
8. Le massif de

maconnerie mesure de 50

à $100 \mathrm{~cm}$ d'épaisseur pour

$3 \mathrm{~m}$ de haut. Le matériau

sableux, jaune à ocre roux

prélevé dans le substrat

environnant, sert à régler

les assises des modules.

De quelques millimètres

d'épaisseur en avant de la

maçonnerie, les joints

peuvent mesurer une

quinzaine de centimètres

en arrière, au niveau des

queues des triangles.
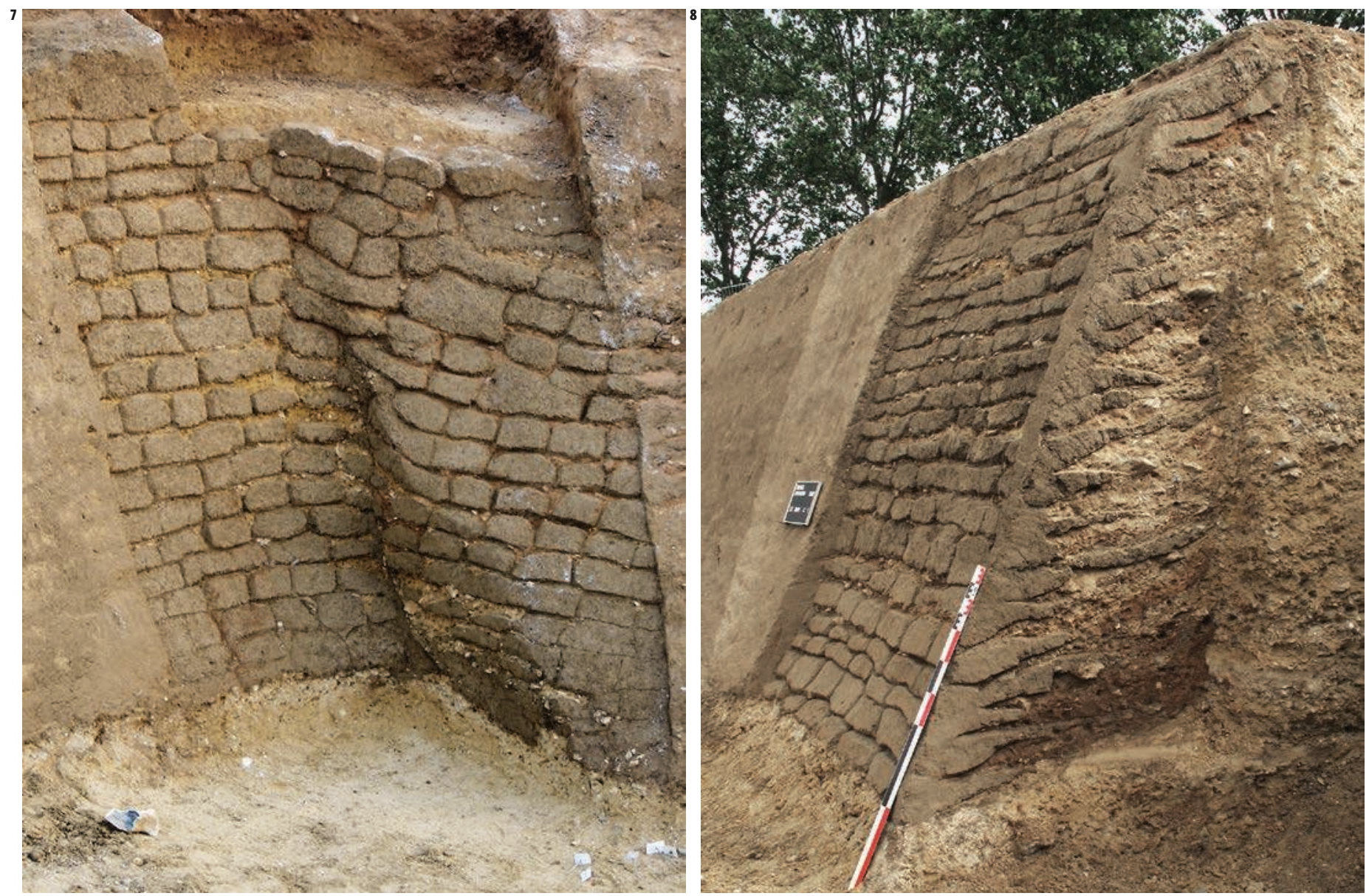
Ville et Vauban indiquent qu'il convient de ficher les mottes vertes à l'aide de piquets, si l'on a pu prendre soin de battre la terre contre les planches pour affermir le rempart. Seul Samuel Marolois précise que la technique peut se matérialiser par l'usage d'une terre grasse limoneuse sans gazon, à condition d'avoir correctement battu et tassé le matériau : "si on peut trouver de la bonne terre grasse E limoneuse, on pourroit (au lieu des gasons) faire une croute de 3 ou 4 pieds, ou plus, le battant bien fort avec un batoir » (Marolois, 1615-1616).

Les études géoarchéologiques ont établi que le matériau a été extrait et mis en œuvre à l'état humide, lors d'une période intermédiaire, c'est-àdire au printemps ou à l'automne (en hiver, il aurait été gorgé d'eau et en été, très sec, il aurait été très difficile à découper). Certains échantillons témoignent d'une sursaturation en eau et d'une hygrométrie qui n'est pas homogène sur l'ensemble du gisement où le matériau est prélevé. La présence de très nombreux petits coquillages lacustres indique un prélèvement à l'emplacement d'une ancienne étendue d'eau, ancien bras mort ou paléochenal. Les modules de terre témoignent d'une forte compaction à l'état moyennement humide qui est accrue en partie basse de la maçonnerie, soumise à un tassement plus important, lié au poids global du massif. L'aplatissement de la porosité des modules de la base de la maçonnerie suggère également une mise en œuvre d'éléments encore humides.

\section{Une adaptation technique issue d'une tradition hollandaise?}

La construction d'un revêtement sur l'escarpe du fossé visait à prévenir son érosion et son effondrement éventuel. Bien conçu, ce revêtement était également une excellente technique de défléchissement des boulets. Le matériau cru absorbait les chocs en réduisant les risques d'éclatement, de dispersion des débris et de propagation des ondes. Le choix de construire un massif sur l'escarpe permettait également de renforcer cette pente du fossé qui soutenaient le poids du rempart de terre. Vauban insiste dans le Traitté de la deffense des places sur ces questions de revêtement en rappelant que l'extérieur des remparts doit être revêtu par de gros murs de maçonnerie ou par des gazonnages ou placages fascinés. Il préconise cette solution dans les lieux où la maçonnerie est chère (par exemple à Colmar et Strasbourg). Il précise également qu'on ne peut attendre une grande résistance de ces revêtements. Ils « ne sont pas faits pour souffrir longtemps le canon mais pour soutenir le rempart et empêcher l'effet d'une escalade ouverte ou dérobée » (Vauban, 17..b).

Le choix du matériau traduit sans doute également l'anticipation par les ingénieurs de la courte durée d'utilisation de l'ouvrage. Si le choix de la terre crue a pu être fait sur certaines fortifications urbaines, elle a souvent été remplacée dans un second temps par la pierre ou la brique, effaçant du même coup les traces de cette technique. Un exemple récent illustre ce propos. Quelques mois après la fouille de Saint-Germainen-Laye, un des membres de l'équipe a pu participer au diagnostic réalisé sur l'ancienne manufacture de tabac de Metz, à l'endroit du bastion Saint-Vincent ${ }^{6}$. Sa vigilance a permis d'observer sur la contrescarpe de ce bastion la même technique de maçonnerie de terre crue. Réalisée par Vauban dans les années 1680, elle précède la mise en place d'une fortification de pierre (Petit, 2013). C'est à ce jour la seule autre occurrence mise en évidence par l'archéologie.

Si cette technique est aujourd'hui méconnue, voire ignorée, elle peut apparaître comme le fruit d'une tradition néerlandaise. L'ingénieur français Marolois, protestant exilé aux Pays-Bas, s'inspirant des techniques locales de fortification, est un des rares à y faire référence. Les choix de fortifier en brique ou en pierre, adoptés par les ingénieurs italiens ou français, convenaient difficilement aux sols humides et marécageux des Provinces-Unies. Trop lourds, les revêtements avaient tendance à s'écarter des fondations et obligeaient à trouver des solutions alternatives (Heuvel, 2007). Les murs en terre se révélèrent moins chers que la brique ou la pierre, et surtout plus légers. Les limons propices à la construction étaient plus abondants et plus simples d'acquisition dans ce paysage de prairies humides. Relativement simples à mettre en œuvre, ils présentaient d'excellentes qualités d'absorption de l'impact des boulets de canons. «Les éléments en terre, souvent utilisés pour renforcer ou pour réparer temporairement les maçonneries endommagées, devinrent de plus en plus permanents. Graduellement, l'intégralité des fortifications de villes fut érigée en terre et en argile, et les expériences évoluèrent d'un processus de recherche par tâtonnements à une méthode plus standardisée, que l'on appela ensuite l'ancienne méthode de fortification néerlandaise » (Heuvel, 2007, p. 43). Champ de bataille de l'Europe, les Provinces-Unies furent également, dès le $\mathrm{XVI}^{\mathrm{e}}$ siècle, un point de convergence de l'ingénierie militaire (Tardy, 2007).

\section{Lépreuve du feu et des intempéries}

L'escarpe maçonnée de Saint-Germain a pu enregistrer, mieux que n'importe quelle autre structure, les dégradations liées aux exercices d'assaut visant à battre le rempart ou certains bastions en brèche, exercices que célèbre la gravure de l'almanach de 1670 .

De manière générale, l'escarpe maçonnée présente un bon état de conservation, favorisé par une excellente résistance du matériau aux contingences extérieures et une période d'exposition sans doute assez courte, puisque le fossé est rebouché à l'issue de la première campagne d'exercice au cours de l'hiver 1669. Des phénomènes d'érosion naturelle, perceptibles dans les coupes stratigraphiques, témoignent ainsi d'une 


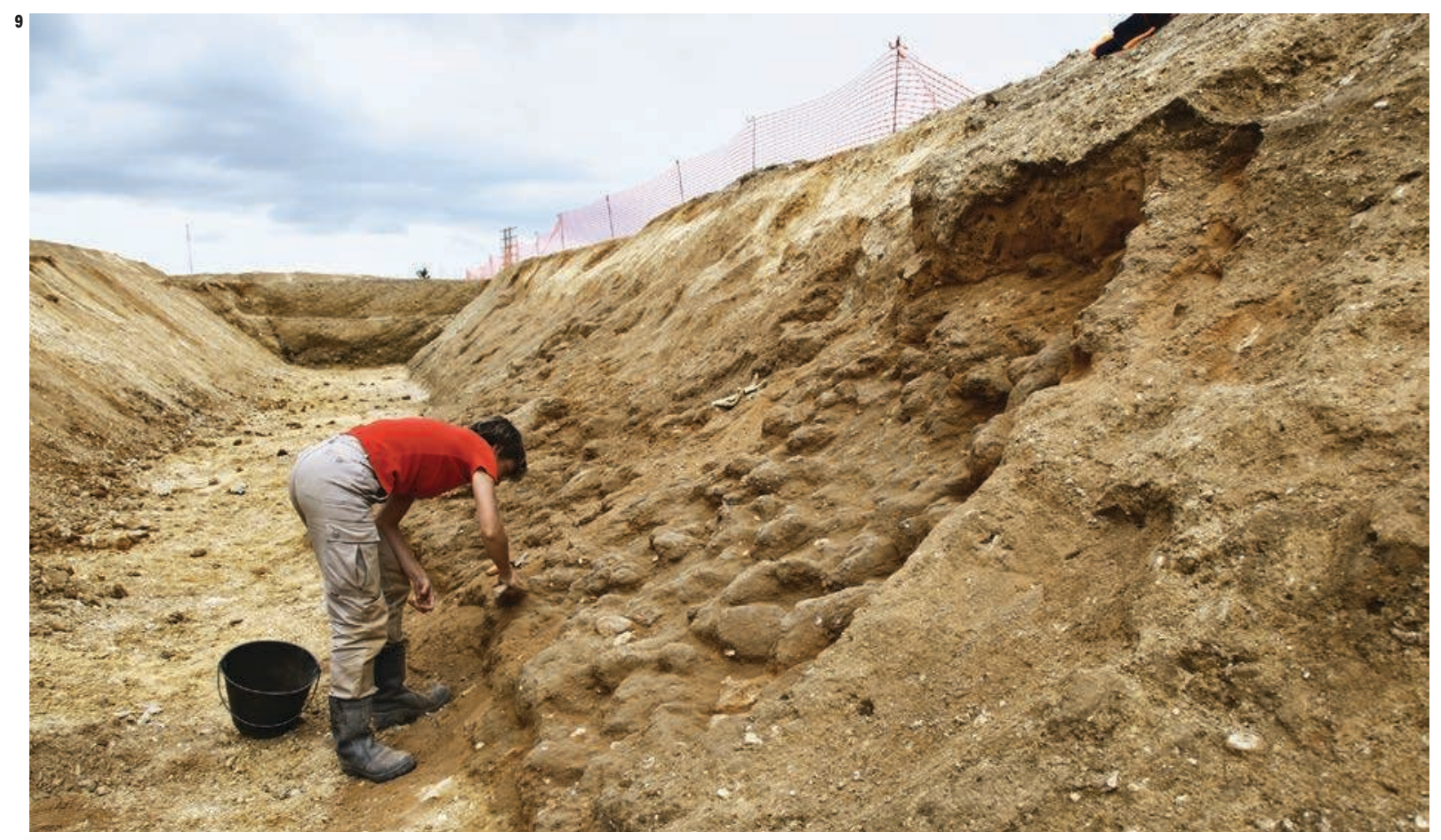

$\cong$

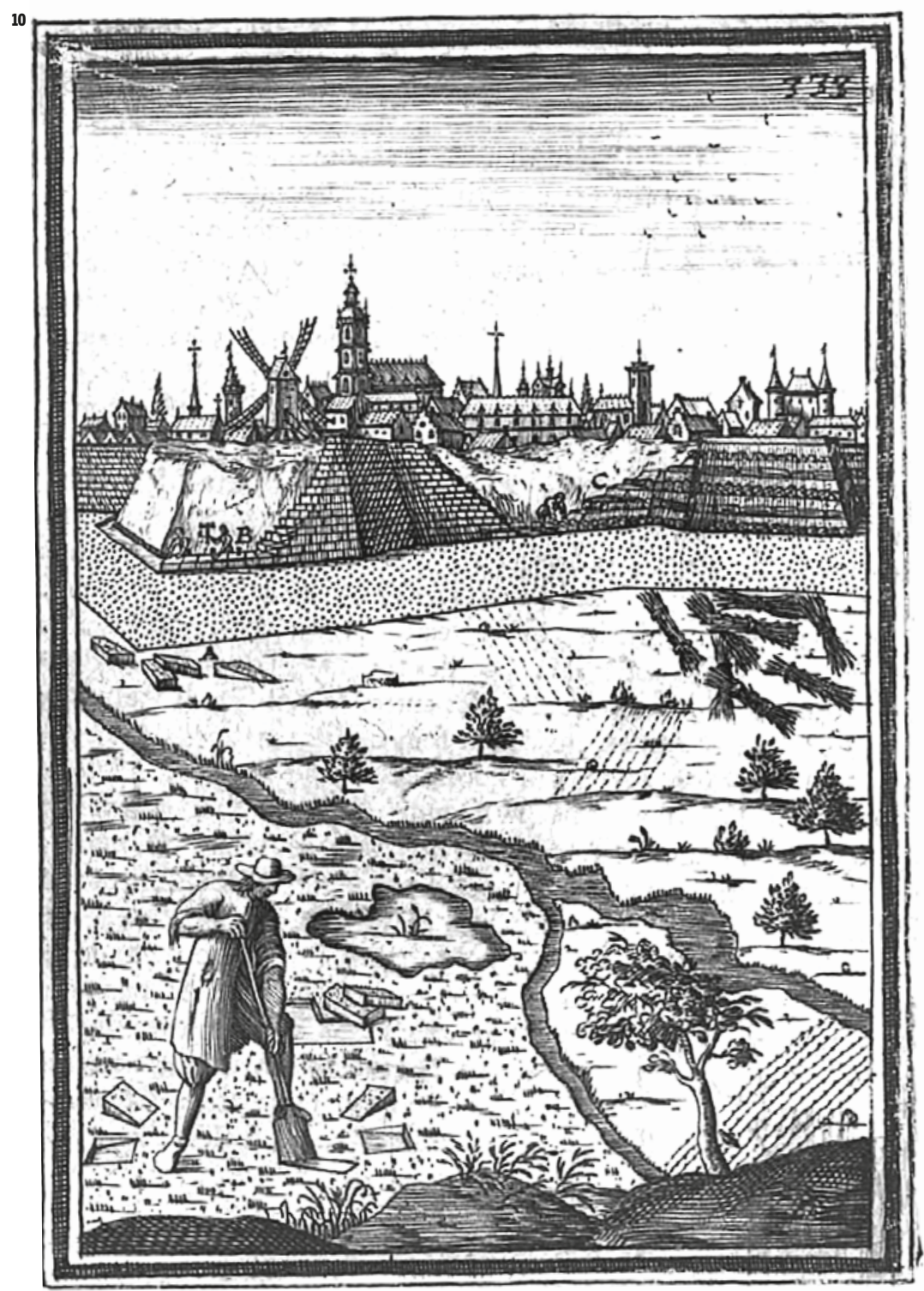

9. Les mottes disloquées offrent un relief marqué

contrastant avec l'aspect

lisse de l'escarpe. Les

modules de terre crue sont

mêlés à de fines couches

de sable indurées qui

témoignent d'un

piétinement des niveaux,

contemporain de

l'utilisation du fossé.

10.Au premier plan, un

personnage débite au

moyen d'une bêche ou d'un

louchet des modules

d'argile triangulaires, dans

une prairie humide,

exprimée par des touffes

d'herbes disparates. Le

chemisage du fossé en

cours de réalisation à

l'arrière-plan

s'accompagne de la mise

en œuvre de fascines dans

un système de litages

successifs entre terre et

bois (Manesson-Mallet,

1684-1685, pl. 333). 
désagrégation limitée des parois sous l'effet des ruissellements. A contrario, le détachement en pans entiers de l'escarpe et l'affaissement consécutif, phénomène observé sur près de $20 \%$ du tracé, pourraient être liés à une action mécanique plus violente. Deux pans argileux dentelés se superposent alors, entre lesquels sont intercalés des volumes variables de sables originaires du massif de maçonnerie. Ce phénomène montre que c'est l'ensemble maçonné (sable et argile) qui se détache et non le revêtement seul : l'assemblage conserve une cohésion certaine. Il s'agit dans la plupart des cas d'un effondrement de la partie supérieure de la maçonnerie qui - détachée en pan - a glissé sur la partie inférieure du revêtement. Nous ne pouvons pas démontrer que ces glissements sont directement liés aux exercices d'assaut ou de canonnage, mais sommes fortement tentés d'aller dans cette direction, notamment en raison de la concentration quasi systématique de ces dommages dans le secteur sud-est du front, alors que toutes les parties sud et ouest sont quasiment épargnées.

Le second phénomène, beaucoup plus anecdotique, concerne le bastion sud-est dont une large section - plus d'une vingtaine de mètres de long - se distingue par son mode de dégradation. Les modules ou mottes n'y montrent plus aucune cohésion et sont indépendants les uns des autres. La paroi détruite est disloquée plutôt que détachée et les mottes semblent désolidarisées les unes des autres par une action mécanique. L'escarpe se présente donc sous une forme complètement éboulée. Il s'agit du seul endroit de la fortification fouillée qui a montré cet état [ill. 9]. Plusieurs sources iconographiques pourraient étayer l'hypothèse d'une brèche ou d'une sape. Dans le chapitre consacré aux mines et sapes de son Traitté de la deffense des places (Vauban, 17..b), Vauban illustre les effets produits par un bombardement ou une mine : on y voit un type d'effondrement et déboulis du revêtement qui ressemble fortement aux observations faites sur le terrain. Les éboulements obtenus par l'explosion permettaient de gravir le talus lors de l'assaut de la place forte. Deux aquarelles - croquis préparatoires de Van der Meulen de 1677 (Paris, Mobilier national et Manufactures nationales des Gobelins, de Beauvais et de la Savonnerie ; Warmoes, 2007) illustrent les effets d'une brèche réalisée sur les fortifications de la citadelle de Cambrai. Si ces fortifications sont d'un autre gabarit que celles du Fort Saint-Sébastien, la représentation met néanmoins en exergue le caractère massif des dégâts généralement obtenus à l'issue d'un bombardement soutenu pendant plusieurs jours ou semaines.

Enfin, bien que plus anecdotique, deux enfoncements du revêtement argileux à deux endroits différents de l'escarpe pourraient correspondre à des impacts de boulets, mais, aussi séduisante qu'elle soit, l'hypothèse reste difficile à démontrer.
Une économie de la terre dans un vaste chantier de terrassement

L'utilisation de terre crue pour les besoins du premier ouvrage fortifié pose de nombreuses interrogations sur l'acquisition de la matière première, son acheminement, la main-d'œuvre nécessaire à l'extraction et à sa mise en œuvre. Toutes ne peuvent pas être réglées à partir des données collectées, mais un certain nombre d'hypothèses et d'estimations peuvent être proposées.

Les propositions de volumes extraits sont fondées sur les calculs de volume et de surface explicités plus haut où nous estimions qu'il fallait compter environ 60 mottes d'argile pour $1 \mathrm{~m}^{2} \mathrm{de}$ maçonnerie, soit environ $0,2 \mathrm{~m}^{3}$ de matériau par $\mathrm{m}^{2}$. À partir de ces données, nous estimons donc que la seule partie fouillée (1130 m linéaire) représentait globalement 203400 mottes d'argile, soit $678 \mathrm{~m}^{3}$ de matériau. Sur la surface totale du fossé, soit $2740 \mathrm{~m}$ de longueur linéaire, l'escarpe représenterait une surface totale de $8220 \mathrm{~m}^{2}$, soit 493200 mottes, pour un volume total estimé à $1644 \mathrm{~m}^{3}$. Si l'on tient compte du fait que ce matériau est prélevé humide, le poids et la masse augmentent d'autant plus. Il faut considérer que chaque seau d'argile de 10 litres, gorgé d'eau, représente un poids de $18 \mathrm{~kg}$, soit un total d'un peu moins de 3000 tonnes à extraire et déplacer.

L'hypothèse de l'extraction des limons dans les horizons alluviaux de la Seine est étayée par l'étude archéogéographique $^{7}$. La partie concave de la plaine alluviale est striée d'anciens chenaux de la Seine qui ont pu constituer d'intéressants gisements. Â une dizaine de kilomètres à l'est du fort, en bordure de la terrasse alluviale, sur l'actuel territoire de la commune d'Achères, de nombreux toponymes indiquent en effet la présence de sites d'extraction de terre : « le Trou du Milieu » et « la Fosse Bidan », « le trou du Nord» sont situés dans un rayon de 3 à $6 \mathrm{~km}$ de l'emprise du fort. Plusieurs gisements ont pu être exploités. Pour extraire les $1600 \mathrm{~m}^{3}$ estimés de matériau nécessaire à la construction, un gisement unique aurait représenté une zone d'extraction de $160 \mathrm{~m}$ de long sur $10 \mathrm{~m}$ de large sur $1 \mathrm{~m}$ de profondeur. Il peut également s'agir d'une zone de $80 \mathrm{~m}$ de long par $20 \mathrm{~m}$ de large sur une profondeur d'1 $\mathrm{m}$. Les solutions sont infinies, mais ce bref calcul montre que la surface exploitée est importante, mais pas démesurée.

Le transport du matériau a pu être facilité par la Seine et assuré par bateau jusqu'au port qui, a priori, se trouvait en aval de lîle d'Herblay, ou par charrois, loués notamment pour les approvisionnements en fourrage et les évacuations des fumures. La proximité entre les gisements potentiels et le site montre que l'on a privilégié une chaîne opératoire courte - où le matériau local est acheminé, à moindre coût, très rapidement après l'extraction - et un temps de stockage réduit dont témoigne l'exploitation du matériau encore humide. Le choix de la terre crue est certainement
7. Réalisée par Émilie Cavanna. 
apparu comme une solution économique et logistique intéressante et a pu en partie guider celui de l'implantation du site dans la terrasse alluviale, la technique étant adaptée aux spécificités du terrain. Alain Manesson-Mallet offre une illustration éloquente sur la nature des sites d'extraction dans un des chapitres de son manuel spécifiquement dédié au chemisage des fortifications (Manesson-Mallet, 1684-1685) [ill. 10].

La question du coût d'un tel chantier et, plus généralement, celle du marché de la terre sont relativement difficiles à aborder. Aucune mention d'un marché passé avec les propriétaires des terrains affectés n'a été retrouvée, pas plus que concernant l'embauche d'artisans ou de journaliers. Il est néanmoins certain que l'exploitation de la terre a fait l'objet d'un marché comme l'approvisionnement en bois, en fourrage ou en viande. Il est peu probable que la couronne ait fait l'acquisition des terrains exploités. Il faut sans doute plutôt envisager - à l'image des propriétés où a été implanté le fort, louées à la marquise de Garennes - un marché de location et un dédommagement des propriétaires, à moins que ces terres n'aient été exploitées par des artisans spécialisés, propriétaires des carrières et dont on aurait loué les services.

Le chantier de construction de l'escarpe maçonnée s'insère évidemment dans un chantier beaucoup plus vaste où sont associés terrassements, travaux de maçonnerie et de charpenterie. L'ensemble représente un défi logistique où la main-d'œuvre et la capacité de recrutement sont des éléments essentiels. L'extraction, le transport, la mise en ouvre de la terre crue et le terrassement des ouvrages nécessitent l'emploi d'une main-d'œuvre qualifiée, voire spécialisée, dont les sources écrites relatives au fort ne font pas mention. Plus d'un siècle avant la création du Génie militaire (1791), Vauban exhortait déjà à la création d'une « troupe à la brouette », de professionnels spécialement dédiés aux terrassements militaires, conscients des problèmes que posait l'usage d'une maind'œuvre non spécialisée. Les soldats constituaient le premier vivier de terrassiers et d'apprentis maçons et étaient encadrés par des professionnels du bâtiment. Il était également d'usage de faire appel à de très forts contingents de paysans enrôlés nécessitant l'organisation de véritables filières de recrutement et de circuits d'approvisionnement à l'échelle suprarégionale. Les hanskers flamands, excellents « remueurs de terre », étaient renommés pour être six fois plus productifs que les paysans français (Destable, 2007, p. 247). Soldats et paysans «maniant la pioche et la pelle, gâchant les mortiers, alimentant les échafaudages et les ateliers en matériaux de toute sorte »s'improvisaient ainsi terrassiers ou maçons (Destable, 2007, p. 245). À titre d'exemple, le siège de Maastricht de 1673 nécessita la réquisition de 20 ooo paysans, on enrôla 16 ooo paysans pour le siège d'Ath en 1697 (Childs, 2001). 60oo paysans furent employés aux travaux de Béthune et d'Arras (Destable, 2007, p. 245).

Afin de motiver les troupes de « remueurs de terre », Vauban insistait pour que les ouvriers soient payés au volume et non à la journée et que la main-d'œuvre soit fermement encadrée. Vauban estime dans le Traité de l'attaque et de la deffense des places qu'un paysan de bonne constitution peut creuser de 1 à 2,5 toises cubes en 7 jours, soit entre 1 à $2,5 \mathrm{~m}^{3}$ par jour et par homme (Vauban, 17..a). Sur la base de ces mêmes chiffres, nous estimons que le terrassement des $57000 \mathrm{~m}^{3} \mathrm{du}$ premier ouvrage a dû prendre quelques semaines entre le début du mois d'avril et la mi-mai 1669, date d'inauguration du fort, soit sur la base d' $1 \mathrm{~m}^{3}$ par jour et par homme : 57 jours à 1000 hommes, 19 jours à 3000 ou 11 jours à 5000 . Sur la base de $2,5 \mathrm{~m}^{3}$ par jour et par homme, il aurait fallu 23 jours à 1000 hommes, moins de 8 jours à 3000 hommes et 4,5 jours à 500o. Les terrassements nécessitaient un outillage abondant composé de pioches, haches, pelles, bêches, louchets, hottes, brouettes et tombereaux. Une mention relative au Fort SaintSébastien révèle que l'arsenal de Paris fournissait les troupes à raison de 500 outils par commande (Archives diplomatiques, Mémoires et documents, 931, fol. 69v). Pour les travaux de la place forte de Dunkerque au printemps 1671, Vauban et Louvois prévoyaient de faire acheminer 8 ooo pelles ferrées et autres louchets de Flandre dont plus de la moitié étaient hors d'usage après trois semaines de travaux (Destable, 2007, p. 248). Certains outils du terrassement, bêches, pelles, pioches et haches ont été mis au jour lors de la fouille. Aussi modestes soient-ils, ils n'en constituent pas moins un témoignage unique des travaux de siège des années 1670 (Hurard, 2014).

L'escarpe maçonnée mise en évidence à SaintGermain constitue, à ce jour, un exemple unique de l'utilisation de terre crue, appliquée à la fortification du XVII ${ }^{\mathrm{e}}$ siècle, qui cache sans doute une réalité largement sous-estimée. Les traités militaires laissent en effet imaginer une utilisation massive au XVII ${ }^{\mathrm{e}}$ siècle, dans le cadre militaire, d'un matériau dont on sous-évalue beaucoup plus généralement l'utilisation dans la construction civile (bauges, pisé, torchis...) et sur lequel il est urgent de se pencher. La maçonnerie de terre crue du Fort Saint-Sébastien illustre la complexité des techniques de poliorcétique moderne où l'issue du siège repose sur la capacité logistique et opérationnelle à assurer l'acquisition et l'acheminement des ressources nécessaires, et est largement conditionnée par les stratégies d'implantation. Au Fort Saint-Sébastien, la répétition de la guerre de siège est un test des capacités logistiques et opérationnelles de l'armée, au moins autant, si ce n'est plus, que de ses capacités tactiques. 


\section{Références bibliographiques}

Archives diplomatiques (La Courneuve), Mémoires et documents, 931 (Affaires intérieures, 191), Mémoires des expéditions des secrétaireries d'État, 1670.

Fournier G., 1648, Traité des fortifications, ou Architecture militaire. Tiré des places les plus estimées de ce temps..., Paris, J. Hénault, 183-[7] p.-[9o] p. de pl.

LA FonTAINE J.-H. DE, 1666, Les fortifications royales, ou Architecture Militaire, par une nouvelle pratique : avec celles de tous les plus excellens autheurs qui ont escrit de cette matiere depuis Henry le Grand, jusqu’à present; E un Traité de l'artillerie \& usage du canon. Ensemble, la Geometrie universelle, E un compendion de perspective; avec la construction des cadrans solaires, l'usage des cadrans analitiques, E autres diverses choses... Paris, E. Loyson, [6]-128-[2] p.

MANeSSON-MALlet A., 1684-1685, Les travaux de Mars, ou l'Art de la Guerre, divisé en trois parties.

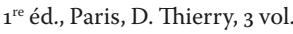

MARolois S., 1615-1616, «Opera mathematica», ou ouvres mathématiques traictans de géométrie, perspective, architecture et fortification, par Samuel Marolois, ausquels sont ajoints les fondements de la perspective et architecture, de $J$. Vredm. Vriese, augmentée et corrigée en divers endroicts par le mesme auteur, Hagae-Comitis, ex off. H. Hondii.

Vauban S. Le Prestre de, 17..a, Traité de l'attaque et de la deffense des places, manuscrit en ligne, [S.l.], [s.n.], 160 f.-33 pl. dépl. Disponible sur http:// cnum.cnam.fr/CGI/redir.cgi?FOLRESQE2 (consulté le 29/03/2015).

Vauban S. Le Prestre de, 17..b, Traitté de la deffense des places, manuscrit en ligne, [S.l.], [s.n.], 216 p.-11 d. p. Disponible sur : http://cnum.cnam. fr/redir?FOLRESQE3 (consulté le 16/05/2014).

VILLE A. DE, 1628, Les fortifications du chevalier Antoine de Ville, contenans la manière de fortifier toute sorte de places... avec l'attaque et les moyens de prendre les places..., Lyon, J. Barlet, [12]-441[12]-[12] p. Disponible en ligne sur : http://cnum. cnam.fr/CGI/redir.cgi?PFOLRESQE2 (consulté le 29/03/2015).
CHILDS J., 2001, La guerre au XVII siècle, Paris, Éd. Autrement («Atlas des guerres »), 224 p.

Destable P., 2007, « La gestion de l'aménagement des places fortes et l'organisation des chantiers de construction ", in Vauban, bâtisseur du RoiSoleil, exposition (Paris, Cité de l'architecture et du patrimoine, 13 novembre 2007-5 février 2008), Paris, Somogy, Cité de l'architecture et du patrimoine, Musée des plans-reliefs, p. 243-249.

Guillaud H., 2003, " Construire en blocs découpés et mottes de gazon », in Chazelles C.-A. DE, KLein A. (DIR.), 2003, Échanges transdisciplinaires sur les constructions en terre crue, vol. 1, Terre modelée, découpée ou coffrée, matériaux et modes de mise en ouvre, Actes de la table-ronde de Montpellier (17-18 novembre 2001), Montpellier, éditions de l'Eśpérou, p. 185-211.

Heuvel C. VAN DEN, 2007, « Les ingénieurs dans la boue : adapter les styles internationaux aux pratiques néerlandaises en matière de fortifications », in Vauban, bâtisseur du Roi-Soleil exposition (Paris, Cité de l'architecture et du patrimoine, 13 novembre 2007-5 février 2008), Paris, Somogy, Cité de l'architecture et du patrimoine, Musée des plans-reliefs, p. 42-47.

HURARD S., 2014, « Le camp d'entraînement des troupes du Roi », in Mousquetaires!, catalogue d'exposition (Paris, Musée de l'Armée, avril-juillet 2014), Paris, Gallimard, Musée de l'Armée, p. 220-221.

Hurard S., Rochart X., 2013, « Le fort SaintSébastien de Saint-Germain-en-Laye : camp d'entraînement des troupes de Louis XIV », in Actes des journées archéologiques d'Île-de-France 2012, Paris, Ministère de la culture, Préfecture de la région Île-de-France, Direction régionale des affaires culturelles, p. 65-78.

Hurard S., Lorin Y., Tixador A., 2014, « Une archéologie de la guerre de siège moderne (XVII ${ }^{\mathrm{e}}-\mathrm{XVIII}^{\mathrm{e}}$ siècles) à l'échelle européenne », Les Nouvelles de l'archéologie, $\mathrm{n}^{\circ} 137$ - novembredécembre 2014, p. 19-24.

Hurard S., Rochart X., 2015, « Régiments de cavalerie des troupes de Louis XIV : Les écuries du fort Saint-Sébastien de Saint-Germain-en-Laye », Archéopages, $\mathrm{n}^{\circ}$ 41, La place du cheval, p. 66-77.

Petit X., 2013, Le site du fort-Saint-Sébastien à SaintGermain-en-Laye : étude géoarchéologique de la dynamique de comblement des fossés défensifs, Mémoire de Master I, archéologie et environnement, Paris, Université Paris I Panthéon-Sorbonne.

TARDY R., 2007, «Les villes fortes bastionnées des pays bas du Nord-Ouest européen ", in Vauban, bâtisseur du Roi-Soleil, exposition (Paris, Cité de l'architecture et du patrimoine, 13 novembre 2007-5 février 2008), Paris, Somogy, Cité de l'architecture et du patrimoine, Musée des plansreliefs, p. 254-258.

WARMOES I., 2007, « Vauban et l'art de la fortification ", in Vauban, bâtisseur du RoiSoleil, exposition (Paris, Cité de l'architecture et du patrimoine, 13 novembre 2007-5 février 2008), Paris, Somogy, Cité de l'architecture et du patrimoine, Musée des plans-reliefs, p. 190-197. 